\title{
Celiac Disease in Bangladesh - Two Case Reports
}

\author{
MD. RUKUNUZZAMAN ${ }^{1}$, ASM BAZLUL KARIM ${ }^{2}$, SM BAQUI BILLAH ${ }^{3}$, MD. ATIAR RAHMAN ${ }^{4}$, MD \\ MAHBUBUL ISLAM ${ }^{5}$, MOHAMMED KAMAL ${ }^{6}$
}

\begin{abstract}
Celiae disease is an immunological disorder precipitated by gluten in genetically susceptible persons. Its prevalence is not known in Bangladesh because of unavailability of its screening test. There is diversity in the presentation of celiac disease. Two children of 5 and 8 years of age who were diagnosed as celiac disease are reported here. One presented typically with chronic diarrhoea \& growth failure. Another child presented with features of chronic liver disease. In both the cases IgA tTGA were positive and duodenal biopsy showed villous atrophy. After diagnosis, both the patients were kept on gluten free diet (GFD). After six months of GFD, IgA tTGA came down to normal in both the cases. They were then given gluten containing diet again \& after few months IgA tTGA again raised in both the cases. Thereafter the cases were finally diagnosed as celiac disease and were advised life long gluten free diet. Celiac disease is not uncommon in Bangladesh and screening test should be done to diagnose or rule out celiac disease when there is a suspicion.
\end{abstract}

Key words: Bangladesh, Celiac disease, Case series, Gluten.

\section{Introduction}

Celiac disease (CD) is an immune-mediated disorder elicited by gluten in genetically susceptible individuals and characterized by the presence of a variable combination of gluten dependent clinical manifestations, celiac disease specific antibodies, HLA-DQ2 or HLA-DQ8 haplotypes and enteropathy. ${ }^{1}$ In Europe and USA; the prevalence is between 1 in 300 to 1 in $80 .{ }^{2}$ Prevalence is not known in Bangladesh due to unavailability of appropriate screening test. There are typical and atypical presentations of CD. Typical features are chronic diarrhoea, weight loss, iron deficiency anemia, etc. Sometimes CD may

1 Associate Professor, Department of Pediatric Gastroenterology \& Nutrition, Bangabandhu Sheikh Mujib Medical University, Dhaka, Bangladesh.

2 Professor \& Chairman, Department of Pediatric Gastroenterology \& Nutrition, Bangabandhu Sheikh Mujib Medical University, Dhaka, Bangladesh

3 Senior Consultant, Department of Pediatric Gastroenterology \& Nutrition, Bangabandhu Sheikh Mujib Medical University, Dhaka, Bangladesh.

4 Assistant Professor, Department of Pediatric Gastroenterology \& Nutrition, Bangabandhu Sheikh Mujib Medical University, Dhaka, Bangladesh.

5 Consultant, Department of Pediatric Gastroenterology \& Nutrition, Bangabandhu Sheikh Mujib Medical University, Dhaka, Bangladesh

6 Professor \& Chairman, Department of Pathology, Bangabandhu Sheikh Mujib Medical University, Dhaka, Bangladesh

Correspondence: Dr. Md. Rukunuzzaman, Email dr.rukon@gmail.com, Phone- 01716835025 present with atypical features like obesity, various form of liver diseases, constipation, arthritis, pubertal delay, dental enamel defects or neuropsychiatric disturbance. ${ }^{3} \mathrm{CD}$ are called silent when patients are asymptomatic but confirmed by small bowel histology. Patients who are previously diagnosed as CD but now normal small bowel histology with gluten containing diet are categorized as latent celiac. Patients with positive genetic background and serology of $C D$ but histologically normal small bowel are called potential celiac. ${ }^{3,4} \mathrm{CD}$ occurs at a higher frequency in children with type 1 diabetes mellitus, Down's syndrome, Turner syndrome, William syndrome, thyroiditis, selective IgA deficiency, etc. ${ }^{4,}{ }^{5}$ Celiac disease is caused by an inflammatory T-cell response to gluten and characterized by the presence of auto-antibodies in blood and histological alterations of the small bowel mucosa. Auto-antibodies are anti-gliadin, anti-reticulin, anti-endomysial and anti-tissue transglutaminase. ${ }^{6}$ Histological examination of small intestine shows villous atrophy, crypt hyperplasia and damage to the surface epithelium. The injury is greatest in the proximal small bowel and extends distally for a variable distance. ${ }^{7}$ In Bangladesh after the introduction of IgA tTGA, as a screening test, few cases of celiac have been identified. Here two cases of celiac disease are described: one presented with chronic diarrhoea with short stature and the other with features of chronic liver disease with portal hypertension. 


\section{Case series}

Case- 1: A 5 year old boy of non consanguineous parents presented with the complaints of recurrent episode of loose motion since 18 months of his age and not growing well for the same duration. Stool was bulky, oily and not mixed with blood. Some times consistency of stool was watery. He had history of recurrent generalized edema on several occasions during last two years. One year back, he had a history of taking anti- tubercular drugs for 6 months.

The child was ill looking, moderately pale, severely stunted (HAZ <-3) and severely underweight (WAZ<$3)$. There was no organomegaly and ascites. Examination of other systems was unremarkable.

Hematological examination shows $\mathrm{Hb}-8.2 \mathrm{gm} / \mathrm{dl}$ with microcytic hypochromic blood picture. Total count of WBC, platelet count and ESR were within normal limit. Stool microscopy was normal and culture was negative of pathogenic organism. Mantoux test was negative. Liver function tests were normal. Barium follow through study of small intestine was unremarkable. Sweat chloride test was done and it was $39.5 \mathrm{mmol} / \mathrm{l}$. Serum IgA tTGA on gluten diet was $122.5 \mathrm{U} / \mathrm{ml}$ (normal up to $50 \mathrm{U} / \mathrm{ml}$ ). Endoscopic duodenal biopsy shows total villous atrophy, crypt hyperplasia and infiltration of chronic inflammatory cells in the lamina propria. The child was provisionally diagnosed as a case of celiac disease and was kept on gluten free diet (GFD). Serum IgA tTGA level came down to normal with improvement of diarrhoea six months after introduction of GFD. Serum IgA tTGA level again rose to $70.0 \mathrm{U} / \mathrm{ml}$ six months after reintroduction of gluten containing diet. Thereafter the case was finally diagnosed as a case of celiac disease and was advised to continue GFD for life long.

Case- 2: A 10 year old boy, $2^{\text {nd }}$ issue of a non consanguineous parents, fully immunized as per EPI schedule, presented with the complaints of haematemesis, chronic diarrhoea and failure to thrive. Several episodes of haematemesis occurred prior to this episode and each time vomitus contained copious amount of fresh blood. Mother also gave the history of recurrent episodes of diarrhoea with pale coloured stool since 8 months of her child's age. Another complain of the mother was that her child was not growing well since his early childhood. The boy was on exclusive breastfed up to 6 months of his age, thereafter complementary feeding was started with mixed rice and pulses. As the child grows, bread, biscuits \& other wheat based foods were added. When wheat based foods were started, diarrhoeal episodes started. On query, there was no history of neonatal sepsis, umbilical infection or catheterization, any history of blood transfusion, surgery, any dental procedure or family history of jaundice. For this reason the child was admitted and treated at different hospitals of Dhaka but his condition did not improve.

On examination, the child was found well alert, afebrile, anicteric and moderately pale. Other vital signs were normal. There was no oedema, dehydration, lymphadenopathy and clubbing. The child was found severely stunted $(H A Z<-3)$. Abdominal examination showed splenomegaly which was $6 \mathrm{~cm}$ from left costal margin along its long axis. Hepatomegaly or ascites was absent. Other systemic examination was normal. From history \& clinical examination, the child was provisionally diagnosed as a case chronic liver disease (CLD) with portal hypertension. Hematological examinations showed $\mathrm{Hb}-7 \mathrm{gm} / \mathrm{dl}$, with microcytic hypochromic blood picture with normal WBC and platelet count. Serum alanine aminotranseferase (ALT) level was raised ( $84 \mathrm{U} / \mathrm{L}$ ), prothrombin time prolonged ( INR 1.6) and serum albumin was $25 \mathrm{gm} / \mathrm{L}$. Ultrasonography of hepatobiliary system showed coarse hepatic echo texture and hepato-fugal blood flow. Serum IgA anti- tissue transglutaminase (tTG) antibody level on normal diet was $126.5 \mathrm{U} / \mathrm{ml}$ (normal up to $50 \mathrm{U} / \mathrm{ml}$ ). Upper $\mathrm{Gl}$ endoscopy showed grade II esophageal varices and endoscopic biopsy from $2^{\text {nd }}$ part of duodenum showed chronic duodenitis with villous atrophy. Haemoglobin electrophoresis was normal. Both hepatitis $B$ and hepatitis $C$ serology were negative. Eye examination was negative for KF ring. Serum ceruloplasmin and 24 hours urinary copper excretion after penicillamine challenge was normal. Anti nuclear antibody and serum $\mathrm{LKM}_{1}$ serology was negative. Serum alpha-1 antitrypsin level was within normal limit. Liver biopsy showed chronic hepatitis with fatty changes, histological activity index( $\mathrm{HAl})$ was 8. The boy was then advised to take GFD and serum IgA tTGA level came down to normal 6 months after introduction of GFD. Later on he was advised to take gluten containing diet again. Serum IgA tTGA again rose to $90.5 \mathrm{U} / \mathrm{ml} 6$ months after re-introduction of gluten containing diet. The boy was then finally diagnosed as a case of CLD due to celiac disease with portal hypertension. He was given life long dietary (GFD) advice and prophylactic propranolol for portal hypertension 


\section{Discussion}

The activity of gluten resides in the gliadin fraction, which contains certain repetitious amino acid sequences that lead to sensitization of lamina propria lymphocytes. Gluten consists of a complex mixture of many gliadin and glutenin polypeptides. Gliadins are monomers, whereas glutenins form large polymeric structures. $^{8}$

There is no sex difference about the occurrence of celiac disease. Both of our cases were male. Celiac disease usually manifests during infancy and before school age but may occur at any age. ${ }^{8,9}$ The present cases presented at 5 year and 10 year of age. Intestinal manifestations are more marked in earlier ages. Gastrointestinal symptoms may be totally missing in older children. ${ }^{8}$ The first case presented typically with chronic diarrhoea and weight loss whereas the $2^{\text {nd }}$ case presented with features of chronic liver disease. Diarrhoea occurs in $87 \%$ and growth failure in $98 \%$ cases in celiac disease. ${ }^{9}$ Diarrhoea results from villous atrophy (decrease in surface area), disaccharidase deficiencies and concomitant infection. Growth failure may be due to malabsorption, chronic diarrhoea and increased catabolism. ${ }^{10,2}$

Wide spectrum of liver injury occurs in celiac disease. The 2 nd case presented with raised ALT with histological features of chronic hepatitis with mild fatty changes. Common liver conditions associated with celiac disease are persistent elevation of serum ALT, reactive hepatitis, autoimmune liver disorders, $\mathrm{NASH}$, acute liver failure, cirrhosis and regenerative nodular hyperplasia. Most of the conditions reverse after introduction of gluten free diet. Liver injury in CD may be due to the facts that increased intestinal permeability of antigens may induce an immune response against self liver protein. About $30 \%$ patients of celiac disease may have some degree of liver involvements. ${ }^{11}$

Celiac patient may present with anaemia. Anaemia was microcytic hopochromic in both of our cases, but anaemia may be dimorphic. Anaemia is due to iron deficiency and is refractory to oral iron therapy. Anaemia results from iron malabsorption from proximal intestine. $^{12}$

Several conditions may mimic CD. These are gastroenteritis, allergic colitis, inflammatory bowel disease (Crohn's disease), giardiasis, immunodeficiency state, bacterial overgrowth syndrome and drugs. ${ }^{2}$ GIT infection in the first case was excluded by stool examination. Allergic colitis was also excluded by dietary modification. Common food allergens like animal milk, egg yolk, and Soya protein were sequentially withdrawn from diet without improvement of the condition. There was also absence of eosinophilic infiltrate in duodenal mucosa. Inflammatory bowel disease was excluded by barium follow through study and colonoscopy \& biopsy from terminal ileum. Giardiasis was excluded by routine stool examination. Immunodeficiency disorders were excluded by doing serum immunoglobulin levels. There were no features of immunodeficiency disorder clinically. Moreover, secondary immunodeficiency disease like human immunodeficiency virus infection is very rare in Bangladesh. In the second case, causes of chronic liver disease were tried to find out. Viral serology for hepatitis $B$ and $C$ were negative. Wilson's disease was excluded by slit lamp examination of eye for K-F ring, serum ceruloplasmin and 24 hours urinary copper excretion after penicillamin challenge. Screening tests for autoimmune hepatitis were also negative. Liver biopsy was suggestive of CLD and it also excluded few causes of CLD like alpha 1 antitrypsin deficiency, autoimmune hepatitis, storage disease etc.

The proper diagnosis of celiac disease is essential to suggest for life long adherence to gluten free diet. Testing for celiac disease should be considered in the following groups of patients: First: those with gastrointestinal symptoms including chronic diarrhoea, malabsorption, weight loss, and abdominal distension. Second: individuals without other explanations for persistent elevation of serum ALT, short stature, delayed puberty, refractory iron-deficiency anemia, etc. Third: patients with type 1 diabetes mellitus or with other autoimmune endocrinopathies, first and second degree relatives of individuals with celiac disease, patients with Turner's or Down's syndrome, patients with juvenile idiopathic arthritis or with Sjogren's syndrome. ${ }^{6}$

In suspected cases evaluation should be started with serological testing. Serum IgA anti-endomysial and anti-tissue transglutaminase antibodies have got equal diagnostic accuracy. Paediatric patients with a IgA tTGA level of $100 \mathrm{U} / \mathrm{ml}$ or more in whom symptoms improve upon consuming a gluten free diet may not need a duodenal biopsy to confirm CD. ${ }^{13}$ IgA tTGA level may be false negative in individual with selective 
IgA deficiency. Antibody levels may remain elevated for varying period of time even up to six months after withdrawal of gluten from diet. ${ }^{14}$

Patients with a positive IgA endomysial or transglutaminase antibody tests should undergo a small bowel biopsy. Exceptions are those who have biopsy-proven dermatitis herpetiformis in whom the diagnosis can be established without a small bowel biopsy. Multiple biopsies should be obtained from the second and third part of duodenum as the distribution of villous atrophy may be patchy. The duodenal mucosa may appear atrophic with loss of folds. The diagnosis is presumptively established when there is concordance between the serologic results and the biopsy findings. It is confirmed when symptoms resolve and serology become normal on a gluten free diet. Demonstration of histological normalization following gluten free diet may not be required. ${ }^{6}$

The initial diagnostic criteria of the European Society of Pediatric Gastroenterology and Nutrition (ESPGAN 1969) required three biopsies. First endoscopic biopsy is required to document a structurally abnormal intestinal mucosa on gluten diet; second biopsy is required to see histological improvement on GFD and third is required to see further villous atrophy after gluten challenge. The revised ESPGAN criteria are more workable and are presently being widely used. The two essential criteria are characteristic small intestinal mucosal changes while on a diet containing adequate gluten and a full clinical remission following GFD. The presence of serological antibodies which disappear while on GFD, further support the diagnosis and should be an essential component of the diagnostic work up. ${ }^{15,} 16$

\section{Conclusion}

It is assumed that celiac disease is present in Bangladesh. As the screening test and biopsy facilities for children are now available, every attempt should be made to diagnose or rule out celiac disease when there is a suspicion. Large community based study especially among risk groups are necessary to know exactly the prevalence of celiac disease in Bangladesh.

\section{References:}

1. Husby S, Koletzko S, Korponay- Szabo IR, Mearin ML, Phillips A Shamir R,etal.European Society for Pediatric Gastroenterology and Nutrition Guidelines for the Diagnosis of Coeliac Disease. JPGN 2012; 54: 136- 160.

2. Soor MR. Gluten Sensative Enteropathy. In: Kliegman RM, Behrman RE, Jenson HB, Stanton
BF, eds. Nelson Textbook of Pediatrics, 18th edition, Philadelphia: Saunders; 2007. p 1591-93

3. Steele R. Diagnosis and management of celiac disease in children. Postgrad Med J 2011; 87: 19-25.

4. Mustalahti K. Unusual Manifestations of Celiac Disease. Indian J Pediatr 2006; 73 (8): 711-716.

5. Mubarak A, Wolters VM, Gerritsen SAM, Gmelig- Meyling F, Ten kate F and Houwen R. A Biopsy Is Not Always Necessary to Diagnose Celiac Disease. JPGN 2011; 52: 554-557.

6. Kelly CP, Lamont JT, Bonis PA. Diagnosis of celiac disease. WWW.uptodate.com 2008, accessed on 21. 03. 2009.

7. Webb C, Halvarsson B, Norstrom F, Myleus A, Carlsson A, Danielsson L et al. Accuracy in Celiac Disease Diagnostics by Controlling the Small bowel Biopsy Process. JPGN 2011; 52: 549-553.

8. Maki M, Lohi O. Celiac Disease. In: Kleinman RE, Sanderson IR, Goulet O, Sherman PM, Mieli-Vergan G, eds. Pediatric Gastrointestinal Disease, $4^{\text {th }}$ edition, Pennysylvania: BC Decker Inc; 2008. P 932- 943

9. Pohl JF, Judkins J, Meihls S, Lowichik A, Chatfield BA and McDonald CM Cystic Fibrosis and Celiac Disease: Both Can Occur Together. Clinical Pediatrics 2010; XX(X): 1- 3.

10. Green PHR, Murray JA. Routine duodenal biopsies to exclude celiac disease? Gastrointestinal Endoscopy 2003; 58 (1): 92-95.

11. Maggiore G, Caprai S. Liver Involvement in Celiac Disease. Indian J Pediatr 2006; 73(9): 809-811.

12. Poddar U, Thapa BR, Singh K. Clinical Features of Celiac Disease in Indian Children: Are They Different Form the West? JPGN 2006; 43: 313-317.

13. Barker CC, Mitton C, Jevon G, Mock T. Can Tissue Transglutaminase Antibody Titers Replace Small- Bowel Biopsy to Diagnose Celiac Disease in Select Pediatric Populations? Pediatrics 2005; 115 (5): 1341-1346.

14. Hill ID, Klish WJ, Hoppin AG. Clinical manifestations and diagnosis of celiac disease in children. WWW.uptodate.com 2008, accessed on 21.03.2009

15. Bhatnagar S, Tandon N. Diagnosis of Celiac Disease. Indian J Pediatr 2006; 73 (8): 703-709.

16. Westerberg DP, Gill JM, Dave B, Diprinzio MJ. Quisel A, Foy A. New Strategies for Diagnosis and Management of Celiac Disease. JAOA 2006; 106(3): 145- 151. 